\title{
Anthony Craig Fischer-Cripps: The Chemistry Companion
}

\author{
Phil Riby
}

Published online: 30 August 2013

(c) Springer-Verlag Berlin Heidelberg 2013

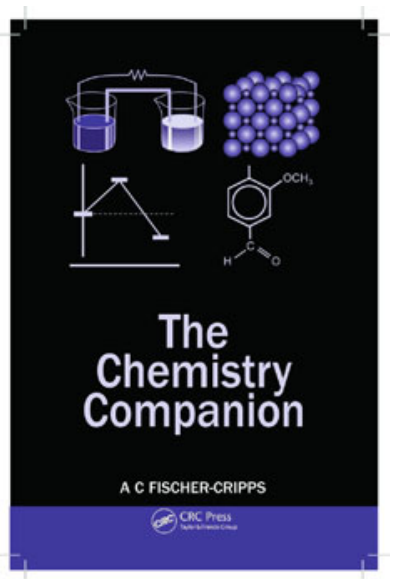

\section{Bibliography}

The Chemistry Companion

A. C. Fischer-Cripps

CRC Press, Boca Raton, FL,

USA, 2012, pp 195

ISBN: 978-1-4398-3088-8

GBP 19.99, USD 31.95

This book says that it 'emphasizes the physics underlying chemistry' and as a result looks at chemistry from a physics point of view. Initially, I was concerned about this but I found the book a good read and it covered the basics from a different angle. Some may be put off by the maths/equations base of some of the sections but if you persist it becomes a good guide to chemistry for people who do not have chemistry as a first science and should not just be regarded as a chemistry book for physicists. I would say it would suit anyone who is interested in an introduction to chemistry. If you are an equation/logic-based individual then it should suit you very well

The book is divided into 12 chapters starting with the structure of matter, chemical components, the periodic

P. Riby ( $\square)$

The School of Pharmacy and Biomolecular Sciences, Liverpool John Moores University, Liverpool, UK

e-mail: P.Riby@ljmu.ac.uk table, chemical bonds, states of matter, chemical thermodynamics, rates of reaction, chemical equilibrium, ionic equilibrium, electronic equilibrium, carbon chemistry and finally a chapter on biochemistry. The book's first chapter starts with the Bohr theory of the atom and the Schrödinger equation which runs the risk of putting off the non-mathematical. Persist, as you will be rewarded with some great explanations of bonding, compound formation, and why states of matter are the way they are. The section on thermodynamics is approached through the application of examples and some simple but effective diagrams. Melting and freezing are considered. The rates of reactions, order, temperature and how catalysis works are considered in the next chapter. Chemical equilibrium is considered using chemical reactions as examples. Le Chatlelier is covered before looking at precipitation. Ionic equilibrium considers acid/bases, $\mathrm{pH}$ and titrations including discussions on the functioning of buffers and indicators. The use of redox reactions, balancing half reactions and the lead/acid battery are covered in electronic equilibrium. Carbon chemistry is covered in the last two chapters. It seems as if the book was complete by Chapter 10 and then two further chapters were added. There is such a lot of material to cover in carbon chemistry and biochemistry that it is unlikely that just two chapters could do it justice and it does not. The areas covered help with naming organic compounds and functional groups and polymers are mentioned. Similarly, the biochemistry section really only introduces the reader to commonly used terminology

Overall, I enjoyed the style of the book as an introduction to chemistry. At under 20 pounds, it is a useful addition for any student who wants to get into chemistry. A Kindle version of the book is available and this should suit the student community 